\title{
Médiévales
}

Langues, Textes, Histoire

51 | automne 2006

L'Occident sur ses marges ( $\left.\mathrm{V}\right|^{\mathrm{e}}-\mathrm{XI}^{\mathrm{e}}$ siècles)

\section{« Poux, vers et vermine » : Étude sémantique sur les parasites des rapaces dans les traductions cynégétiques françaises}

"Lice, Worms and Vermin ": a Semantical Study of the Names of the Birds of Prey's Parasites in Cynegetic Texts translated from Latin to French.

\section{An Smets}

\section{OpenEdition}

\section{Journals}

Édition électronique

URL : https://journals.openedition.org/medievales/1465

DOI : 10.4000/medievales. 1465

ISSN : $1777-5892$

\section{Éditeur}

Presses universitaires de Vincennes

\section{Édition imprimée}

Date de publication : 1 décembre 2006

Pagination : $97-118$

ISBN : 978-2-84292-193-4

ISSN : 0751-2708

\section{Référence électronique}

An Smets, « «Poux, vers et vermine » : Étude sémantique sur les parasites des rapaces dans les traductions cynégétiques françaises », Médiévales [En ligne], 51 | automne 2006, mis en ligne le 27 mars 2009, consulté le 23 avril 2022. URL : http://journals.openedition.org/medievales/1465 ; DOI https://doi.org/10.4000/medievales.1465

Ce document a été généré automatiquement le 23 avril 2022

Tous droits réservés 


\title{
«Poux, vers et vermine » : Étude sémantique sur les parasites des rapaces dans les traductions cynégétiques françaises
}

\author{
"Lice, Worms and Vermin": a Semantical Study of the Names of the Birds of \\ Prey's Parasites in Cynegetic Texts translated from Latin to French.
}

\section{An Smets}

1 Plusieurs études ont déjà insisté sur l'importance des traductions dans le développement du lexique scientifique en langue vernaculaire, et des procédés employés par les traducteurs pour y introduire de nouveaux mots et de nouveaux sens ${ }^{1}$. Nous analyserons ici ce phénomène dans un type de texte particulier, les traités médiévaux consacrés à la chasse au vol, et dans un champ sémantique spécifique, les noms de parasites. Ce choix se justifie par le fait que les parasites sont des maux fréquemment cités dans ces ouvrages ${ }^{2}$. L'examen tiendra également compte du degré d'exactitude des traductions et de la provenance des noms français employés par les traducteurs.

2 Pour cette analyse, nous nous sommes fondés sur les traductions en ancien et en moyen français des traités de fauconnerie latins. Ce corpus contient les textes suivants ${ }^{3}$ :

\begin{tabular}{|l|l|l|}
\hline Sigle $^{4}$ & Texte (latin) & Datation (latine et) française \\
\hline \hline \multirow{2}{*}{ AGfr } & Albert le Grand, De falconibus ${ }^{5}:$ & $(1240-50)$ \\
& - Des faucons I (AGfr I) & - Fin du XIV siècle \\
& - Des faucons II (AGfr II) & - Milieu du Xve siècle \\
& - Des medecines des faucons (AGfrm) & $-2^{\mathrm{e}}$ moitié du XV siècle \\
& - Des faucons III (AGfr III) & - début du XVI siècle \\
\hline
\end{tabular}




\begin{tabular}{|c|c|c|}
\hline Alfr & Alexander medicus ${ }^{6}$ & $\left(\mathrm{XII}^{\mathrm{e}}\right.$ siècle $) \mathrm{XIV}^{\mathrm{e}}$ siècle \\
\hline Dfr & Dancus rex $I$ et $I I^{7}$ & $\begin{array}{l}\text { (xII }{ }^{\mathrm{e}} \text { siècle) I : } 1284, \mathrm{II}: 2^{\mathrm{e}} \text { moitié } \mathrm{du} \mathrm{XV}^{\mathrm{e}} \\
\text { siècle }\end{array}$ \\
\hline Ghfr & Ghatrif, traduction par Daniel Delau ${ }^{8}$ & (vers 1240) 1249-1272 \\
\hline Gufr & Guillelmus falconarius ${ }^{9}$ & (XII ${ }^{\mathrm{e}}$ siècle) 1284 \\
\hline Mfr & Moamin, traduction par Daniel Delau ${ }^{10}$ & (vers 1240) 1249-1272 \\
\hline Pfr & $\begin{array}{l}\text { Epistola Aquile, Symachi et Theodotionis ad } \\
\text { Ptolomeum regem }^{11}\end{array}$ & $\left(\mathrm{XII}^{\mathrm{e}}\right.$ siècle $) \mathrm{XIV}^{\mathrm{e}}$ siècle \\
\hline
\end{tabular}

3 Les sources latines de ces traductions comptent parmi les plus importants textes de la littérature cynégétique ${ }^{12}$. Certains sont à rattacher à la cour normande de Sicile, à savoir Alexander medicus et la suite Dancus rex-Guillelmus falconarius. Ces deux traités se trouvent souvent ensemble dans les manuscrits, et le Dancus rex, surtout, a été largement diffusé, et traduit en plusieurs langues vernaculaires ${ }^{13}$. Leurs auteurs supposés sont fictifs, comme ceux qu'invoque un autre texte de la même époque, à savoir l'Epistola Aquile, Symachi et Theodotionis ad Ptolomeum regem. Parmi les traités latins $\mathrm{du}$ XIII ${ }^{\mathrm{e}}$ siècle, deux ont une origine orientale (Ghatrif et Moamin), et ont été mis en latin à la cour de l'empereur Frédéric II ${ }^{14}$. L'autre texte du XIII ${ }^{\mathrm{e}}$ siècle, le De falconibus d'Albert le Grand, fut inséré plus tard dans l'ouvrage plus large De animalibus du même auteur, et connut ainsi une importante diffusion.

4 Quant aux traductions françaises, elles sont anonymes, à l'exception de celles du Ghatrif et du Moamin, réalisées toutes deux par Daniel Delau ou Deloc à la demande de Henri (Enzo) de Sicile (1224-1272), fils naturel de Frédéric II. Malheureusement, nous ne possédons pas de données semblables sur l'origine des autres versions françaises.

5 Après une présentation de la terminologie latine, nous aborderons le vocabulaire français, et cette analyse comportera deux phases, à savoir un examen terminologique suivi d'une étude des choix de traduction. Mais deux remarques s'imposent au préalable : tout d'abord, il convient de faire une distinction entre les endoparasites ou vers intestinaux qui se trouvent à l'intérieur du corps, et les ectoparasites dont les plus connus sont les poux et les teignes. Deuxièmement, pour des règles inhérentes à l'analyse lexicographique, nous mentionnons les termes à examiner au singulier, bien qu'ils figurent presque toujours au pluriel dans le corpus.

La terminologie latine

6 En ce qui concerne les ectoparasites, notre corpus se limite aux poux et aux teignes; les premiers se présentent dans tous les textes, à l'exception d'Alexander medicus ${ }^{15}$, alors que les teignes figurent chez Albert le Grand, et dans Dancus rex, Guillelmus falconarius et l'Epistola. La terminologie latine se limite à pediculus et tinea ${ }^{16}$. Toutefois, M.-D. Glessgen mentionne que d'autres (ecto-)parasites peuvent se cacher sous le nom de "pou", étant donné que les différents parasites étaient rarement visibles à l'œil nu et donc difficiles à distinguer ${ }^{17}$. Cette remarque vaut probablement aussi pour " teigne». Le substantif pediculus, qui comme sa variante peduculus est un diminutif de pedis (« pou »), 
a fini par supplanter la forme originelle, de sorte que dès l'époque impériale, pediculus est la seule forme attestée ${ }^{18}$. Dans le manuscrit I de Moamin ${ }^{19}$, on rencontre également, une seule fois, la paraphrase infirmitatis pediculorum (II.23.32). Quant au terme tinea, il vient de la médecine humaine, où il désignait généralement soit la « rogne ou gale qui s'attaque à la tête ", soit « un "ver" ou parasite des tissus " ${ }^{20}$. Il est également attesté dès la période du latin classique ${ }^{21}$.

7 La variété lexicale est plus grande pour les endoparasites. Pour les désigner, la médecine humaine emploie les substantifs lumbricus, et, dans une moindre mesure, vermis $^{22}$. De leur côté, les traités cynégétiques latins de notre corpus utilisent - selon l'ordre alphabétique - les termes suivants: aculeus (AG), acus (Al), anguila (AG), lumbricus (AG, D, Gh, Gu, M) et vermis (Gh, M), auxquels il faut encore ajouter punctura $(\mathrm{AG}, \mathrm{Al})$, qui sert à expliquer d'autres termes de cette liste. L'identification exacte de ces différents noms n'est pas aisée. Comme l'a remarqué Baudouin Van den Abeele, «il serait étonnant que le foisonnement des appellations corresponde à autant de catégories de parasites, mais en l'absence de descriptions, il est impossible de se prononcer $"{ }^{23}$.

8 Comme en médecine humaine, le terme le plus fréquent de notre corpus est lumbricus, qui se présente dans cinq traités différents. L'étymologie en est incertaine, mais il connaissait un usage courant dès la période du latin classique, aussi bien dans le sens de "ver de terre" que dans celui de "ver intestinal " ${ }^{24}$. Ensuite vient vermis, qui se présente dans les traductions latines de Ghatrif et de Moamin, qui sont faites par le même traducteur et emploient donc la même terminologie. Le substantif vermis, signifiant « ver », est également déjà attesté en latin classique ${ }^{25}$. Selon J. N. Adams ${ }^{26}$, vermis est le terme général pour désigner les vers qui infectent les chevaux ${ }^{27}$.

9 Ces deux termes, lumbricus et vermis, sont clairement les termes de base ou prototypiques ${ }^{28}$, employés dans tous les traités sauf Alexander medicus. Dans notre corpus, ils désignent tous deux les vers intestinaux, un sens attesté dans les dictionnaires pour lumbricus, alors qu'ils donnent généralement une acception plus large pour vermis ${ }^{29}$. En fait, le choix entre vermis et lumbricus correspond à une différenciation entre un terme courant et un terme déjà plus spécifique, voire plus scientifique. En effet, même si lumbricus figure dans quelques ouvrages classiques non scientifiques ${ }^{30}$, ce n'est pas un hasard si les deux exemples médiévaux cités par R. Latham ${ }^{31}$ proviennent de l'Alphita (un glossaire médical et botanique) ${ }^{32}$ et de l'encyclopédie De proprietatibus rerum de Barthélemy l'Anglais ${ }^{33}$.

10 Ces termes généraux s'opposent à aculeus, acus et anguilla, qui expriment d'après nous des tentatives pour apporter une première distinction parmi les différents vers intestinaux. Que cela ne fût pas chose aisée à une époque sans microscopes est indiqué par le fait que ces trois termes ne connaissent chacun qu'une seule occurrence, qui figure en outre chaque fois à l'intérieur d'une séquence explicative. Le cas le plus clair se trouve chez Albert le Grand: anguillae, hoc est lumbrici longi. Le substantif anguilla, déjà attesté lui aussi en latin classique ${ }^{34}$, y constitue un remplacement d'Albert le Grand pour angulia qui figure dans sa source, Gerardus falconarius ${ }^{35}$. Si angulia semble assez rare - nous ne l'avons trouvé dans aucun des dictionnaires consultés -, la solution de rechange ne semble pas très commune non plus, en tout cas pas dans ce sens. En effet, le MLW ${ }^{36}$ mentionne comme seul exemple d'anguilla signifiant "ver" l'occurrence en question. La rareté du sens pourrait expliquer pourquoi Albert le Grand, quand il reprend dans le même paragraphe la cause du mal, recourt de nouveau 
à lumbrici et non à anguillae (XIX.7). Quant à l'identification, Albert le Grand nous aide par l'insertion de la glose hoc est lumbrici longi, il s'agit donc de vers plus grands. Selon Baudouin Van den Abeele ${ }^{37}$, ce sont probablement des vers du type des Ascaridia, de trois à quinze centimètres et donc visibles à l'œil nu ${ }^{38}$.

Les deux autres noms, à savoir acus et aculeus, sont clairement liés. Leur sens est contenu dans la racine ac-, qui se présente dans toute une série de mots « dont le sens propre ou dérivé, physique ou moral, est demeuré en général proche du sens originel », c'est-à-dire "être piquant, aigu, pointu » ${ }^{39}$. Plus particulièrement, acus signifie généralement " aiguille » et aculeus « aiguillon, épine " ${ }^{40}$. Contrairement à ce qui arrive dans la langue vernaculaire, où le nom français aiguille désigne au moins jusqu'au $\mathrm{XIX}^{\mathrm{e}}$ siècle un type de ver ${ }^{41}$, ce sens d'acus semble en latin restreint au traité d'Alexander medicus ${ }^{42}$. Les auteurs semblent plutôt préférer aculeus comme désignation des «aiguilles» en tant que vers intestinaux, étant donné que ce terme figure non seulement chez Albert le Grand, mais aussi chez Adélard de Bath ${ }^{43}$ et dans le Tractatus de austuribus, source d'Albert le Grand dans ce passage ${ }^{44}$. Mais comme ce sens n'était probablement pas très fréquent non plus ${ }^{45}$, Albert a inséré aculeus dans une séquence plus longue, à savoir le binôme aculei sive puncturae acutae (XXI.9). Cette solution ressemble beaucoup à la glose qui sert à expliquer acus dans Alexander medicus, c'est-àdire punctura quod acus appellamus ( $\mathrm{Al}$ 9). Le substantif punctura, qui est dérivé du verbe pungere, apparaît pour la première fois, selon A. Ernout et A. Meillet ${ }^{46}$, dans les ouvrages du médecin Celse ( $\mathrm{I}^{\mathrm{er}}$ siècle après $\mathrm{J} .-\mathrm{C}$.), bien qu'il ne figure pas dans l'index des mots établi par William Richardson ${ }^{47}$. Du Cange ${ }^{48}$ en donne cependant aussi un sens médical, à savoir " point de côté " : en relation avec les vers, il s'agit donc de deux maux différents mais présentant des symptômes semblables ${ }^{49}$.

La terminologie françaiseAnalyse terminologique

En ce qui concerne les ectoparasites, comment les traducteurs ont-ils rendu pediculus et tinea ? La traduction prototypique de pediculus est pou ${ }^{50}$, avec des graphies parfois fort différentes, même à l'intérieur d'un seul texte ${ }^{51}$. Le substantif en question est déjà attesté dès le $\mathrm{XII}^{\mathrm{e}}$ siècle, d'abord sous les formes püil et peoil ${ }^{52}$. Signalons que Mfr emploie parfois des paraphrases pour rendre pediculus : enfermité de peoil (II.46.26 et II. 93.1) et maladie qi vient par occaison de peoil (III.15.1). Le paragraphe II.46.26 du texte français correspond au paragraphe II.23.32 qui présente dans le manuscrit I la paraphrase infirmitatis pediculorum, alors que le manuscrit $\mathrm{T}$ mentionne la séquence pediculorum natorum au paragraphe $\mathrm{III} .15^{53}$. Toutefois, dans le passage latin correspondant au paragraphe II.93.1, aussi bien I que $\mathrm{T}$ utilisent le nom simple pediculus.

13 À côté du substantif peoil, plusieurs traducteurs recourent en outre à l'adjectif peoillos, qui se présente lui aussi avec différentes graphies ${ }^{54}$, et est attesté dès le XII ${ }^{\mathrm{e}}$ siècle ${ }^{55}$.

Un autre substantif se présente dans AGfr II, d'abord dans le binôme poes ou telle vermine et ensuite de façon autonome. Le terme vermine peut être considéré comme un hyperonyme ${ }^{56}$ de pou, étant donné que ce nom désigne l'ensemble des insectes parasites. La traduction rend donc compte du phénomène en vertu duquel différents parasites peuvent se cacher sous les noms latins correspondant à « pou » ou « teigne ». Le substantif vermine, issu de vermis, apparait également dans le domaine français au $\mathrm{XII}^{\mathrm{e}}$ siècle ${ }^{57}$. différentes : taigne(s) dans AGfrm et Dfr II, t(e)igne dans AGfr I et III et tine(s/z) dans AGfr 
II, AGfrm, Dfr I et Gufr I. Ce substantif, attesté dès le XII ${ }^{\mathrm{e}}$ siècle, existe toujours ${ }^{58}$, mais le sens qu'il revêt dans les traités cynégétiques médiévaux est maintenant considéré comme archaïque. Actuellement, ce nom ne désigne plus les parasites mêmes, mais « une maladie parasitaire de la peau et des phanères qui atteint les oiseaux détenus dans de mauvaises conditions ", qui se manifeste dans une phase avancée sous la forme d'ulcérations des mains et des ailes ${ }^{59}$.

16 À côté de la traduction par le nom simple teigne, relevons le maintien du terme latin dans la table de Dfr I et plusieurs paraphrases contenant soit le nom latin, soit le nom français. Ainsi, le traducteur d'AGfr II a utilisé d'abord deux fois une paraphrase dans laquelle il désigne la teigne comme une espèce de ver, ce qui est erroné : ung ver que on apelle tynia (XVIII.15) et vers que l'on appelle tynes (XIX.10) ${ }^{60}$. C'est seulement pour les deux dernières occurrences qu'il s'est ensuite servi uniquement du nom français. La situation est encore différente dans AGfrm, parce que, pour la première occurrence, le traducteur y utilise un trinôme, à savoir taigne, rougne ou autre infection sur le cuir. À côté $\mathrm{du}$ terme prototypique, le trinôme contient les noms rougne et infection. Le premier substantif, dérivé de *ronea (" action de gratter »), est attesté dès le XII ${ }^{\mathrm{e}}$ siècle avec le sens de "gale invétérée " ${ }^{61}$. L'autre substantif, infection, est un terme savant plus général, issu du latin infectio, dont le sens premier était « action de teindre » (< inficere, " recouvrir de, teindre »). Chez les auteurs chrétiens, ce substantif a ensuite acquis l'acception de "souillure", qui est aussi passée en français, où les premières attestations, de la fin du XIII ${ }^{\mathrm{e}}$ siècle, se situent également dans le domaine moral. Mais, à partir du XIV ${ }^{\mathrm{e}}$ siècle, le substantif infection s'emploie aussi dans un contexte médical ${ }^{62}$. Comme c'était le cas avec le binôme contenant le nom vermine qui rendait pediculus, le trinôme analysé ici indique qu'on peut accorder un sens plus large à tinea.

17 Enfin, les traducteurs optent parfois pour d'autres termes français. Ainsi, le traducteur d'AGfr I emploie, à côté de teigne, également lentes, et celui de l'Epistola présente comme traduction unique migne. Le nom lente («œuf des poux») vient du latin lens et attesté dès le $\mathrm{XII}^{\mathrm{e}}$ siècle ${ }^{63}$. La traducteur a ici confondu deux types d'ectoparasites, et la traduction doit être considérée comme incorrecte. En revanche, l'emploi du nom migne («ver rongeur, mite») dans la traduction de l'Epistola est correct, parce que c'est un synonyme de teigne. Ce substantif apparaît pour la première fois dans les Miracles de Nostre Dame de Gautier de Coinci vers $1224^{64}$, mais ne semble pas avoir été très fréquent. En effet, le TL ${ }^{65}$ mentionne seulement l'exemple cité ci-dessus, le FEW ${ }^{66}$ signale une seule occurrence chez Molinet (entre 1464 et 1506), F. Godefroy ${ }^{67}$ compte également un seul exemple plus ou moins contemporain du précédent, mais dont le sens figuré lui échappait ${ }^{68}$, et le BLMF n'en contient pas du tout. La fréquence peu élevée de mi(g)ne est probablement due à la concurrence avec le substantif mite, qui apparaît également au XIII ${ }^{\mathrm{e}}$ siècle et qui est toujours considéré comme un synonyme de teigne ${ }^{69}$.

Pour désigner les endoparasites, la terminologie latine était caractérisée par une grande variété, et les traductions présentent encore plus de variantes. Ainsi, pour rendre lumbricus, les traducteurs recourent à ver (AGfr I, AGfrm, Dfr II), lombrice (Ghfr, $\mathrm{Mfr}$ ), filandre (AGfr III), esguille (AGfr III) et même à flux de ventre qui le blece (AGfr II), ou conservent le terme latin (Dfr I, Gufr I), soit six solutions différentes pour un seul nom latin. Le substantif ver, dérivé du latin vermis et donc sans aucun rapport formel avec lumbricus, est employé par trois traducteurs. Il est attesté dans le domaine français dès le $\mathrm{x}^{\mathrm{e}}$ siècle sous la forme de verm ou verme et avec le sens de «larve de certains insectes 
qui ronge le bois ». Vers le milieu du XII ${ }^{e}$ siècle apparaitt le sens de "lombric terrestre ", mais selon les dictionnaires ${ }^{70}$, c'est seulement autour de 1380 que ce nom signifie " parasite en forme de ver qui se développe dans le corps de l'homme et des animaux ». Dans cette optique, l'occurrence dans AGfr I peut figurer parmi les premières attestations de ce sens, mais il faut reculer cette datation d'au moins un siècle, parce que les traductions Ghfr et Mfr, qui datent déjà du troisième quart du XIII ${ }^{\mathrm{e}}$ siècle, emploient également verm dans l'acception de « ver intestinal ».

Pour lumbricus, Ghfr et Mfr emploient lombrice. Cette fois-ci, le lien avec le nom latin semble évident et $\mathrm{H}$. Tjerneld mentionne en effet que le manuscrit $\mathrm{X}$ emploie lumbricus ${ }^{71}$. Toutefois, on ne voit pas clairement quel manuscrit a servi de modèle au traducteur et on ne peut donc pas se prononcer sur l'éventuel lien entre le texte latin et la traduction, étant donné que le manuscrit $\mathrm{C}$ porte vermis ${ }^{72}$, tout comme les manuscrits $\mathrm{T}$ et $\mathrm{I}^{73}$. Le substantif français est attesté dès la fin du XII ${ }^{e}$ siècle, mais dans la première mention connue, il a le sens de "vibrion qui se développe dans les cadavres en décomposition ${ }^{74}$, et le traducteur Daniel Delau peut donc ici aussi être le premier auteur à employer ce terme dans le sens de "ver intestinal » ${ }^{75}$. Par rapport à la situation latine, nous constatons ici une inversion de la fréquence d'emploi, étant donné que lumbricus était plus fréquent que vermis, mais lombrice est clairement moins utilisé que ver ${ }^{76}$.

Dans AGfr III, nous trouvons encore deux autres noms, à savoir filandres et esguilles. Le substantif filandre n'a à nouveau aucun rapport formel avec le nom latin, mais est dérivé du latin filum («fil») et apparaît vers la fin du xIII siècle avec le sens de " ficelle " ${ }^{77}$. Un siècle plus tard, les Livres du roy Modus et la royne Ratio présentent les premières occurrences de filandres avec le sens de "petits vers intestinaux des oiseaux de proie ", qui existe toujours ${ }^{78}$. Selon Hubert Beaufrère ${ }^{79}$, il s'agit des vers que l'on trouve dans les émeuts, probablement des capillaires. Quant à esguilles, ce nom ne rend pas vraiment lumbricus, mais reprend le terme anguilla qui figure au début du même paragraphe que l'occurrence de lumbricus en question. Nous le commenterons donc plus en détail ci-dessous, à propos des traductions d'anguilla.

21 Enfin, la traduction est complètement différente dans AGfr II, qui rend lumbricus par flux de ventre qui le blesse. La séquence flux de ventre (du latin fluxus, « écoulement ») est assez fréquente dans les traités médicaux du Moyen Âge et indique, dès le xIII ${ }^{\mathrm{e}}$ siècle, la diarrhée ou la dysenterie ${ }^{80}$. Ces symptômes n'apparaissent pas dans les traités latins ${ }^{81}$, mais C. Bougerol affirme que la capillariose et l'ascaridiose, infections dues à deux sortes d'endoparasites, peuvent provoquer la diarrhée ${ }^{82}$. Le second traducteur a donc opté pour une traduction métonymique, en remplaçant la cause par l'effet. La présence du flux de ventre peut s'expliquer par le texte latin, qui mentionne que les vers apparaissent parfois dans les déjections ${ }^{83}$. Toutefois, si un lecteur non averti, ne disposant pas du texte latin, ne peut se fonder que sur la description de ce symptôme (flux de ventre), où tout rapport avec les vers a disparu, il risque de se tromper sur la cause exacte du mal, c'est pourquoi nous considérons cette traduction comme incorrecte.

Les autres noms latins, qui semblent désigner des types plus spécifiques, figurent toujours dans une combinaison, à l'image de la séquence « anguillae, hoc est lumbrici longi » (AG), dont AGfr III donne une traduction littérale, à savoir esguilles, qui sont vers longs. À première vue, anguilla et esguille, l'actuel aiguille, se ressemblent. Le prétendu rapport formel entre ces deux noms pose cependant quelques problèmes. Le premier se 
situe au niveau étymologique, parce qu'aiguille ne remonte pas à anguilla ( $>$ anguille), mais au latin tardif acucula («aiguille de pin»), un substantif lui-même fondé sur l'adjectif acus (" aigu ») ${ }^{84}$. Le second problème est d'ordre sémantique. Le nom français est attesté pour la première fois chez Gace de la Buigne ${ }^{85}$ et désigne ainsi dès le troisième quart du XIV ${ }^{e}$ siècle « une maladie des faucons causée par de petits vers courts (en forme d'aiguilles) qui s'engendrent dans leur chair ${ }^{86}$. Selon H. Beaufrère ${ }^{87}$, il s'agit de " vers microscopiques qui se logent sous la peau de l'oiseau » et dont la nature exacte est difficile à déterminer. Toutefois, même si leur nature exacte est difficilement identifiable, l'élément microscopique exclut toute identification avec le ver anguilla qui était caractérisé comme un lumbricus longus. La traduction d'aiguille pour anguilla doit donc être considérée comme incorrecte. Étant donné le grand foisonnement des noms, on pourrait cependant comprendre la confusion du traducteur, d'autant plus que le sens de « ver " pour anguilla semble propre à Albert le Grand, ce qui aurait pu faciliter le passage vers le terme connu (aiguille) ${ }^{88}$. En outre, la même confusion se retrouve dans AGfrm, qui rend la paraphrase latine par aguilles, c'est a dire petits vers longs qui tiennent es rains. La séquence française est manifestement contradictoire, mais cette contradiction pourrait éventuellement être expliquée par la tradition manuscrite. En effet, il n'est pas exclu que dans une copie antérieure au présent codex, le texte français portait anguilles, c'est a dire vers longs qui tiennent es rains, avec un tilde pour le $<\mathrm{n}>$ d'anguilles. Dans une copie postérieure, le scribe aurait oublié de mettre le tilde, donnant naissance à la forme aguilles. Comme les aguilles (ou aiguilles) sont de petits vers, un autre scribe aurait inséré plus tard cet adjectif, sans se rendre compte ou en tout cas sans se soucier de la contradiction qu'il introduisait de cette façon dans le texte. Une telle hypothèse est séduisante mais il faut rester prudent, étant donné que nous n'avons pas conservé d'autres copies d'AGfrm qui pourraient la confirmer. De son côté, le traducteur d'AGfr I a évité tout problème terminologique en ne reprenant que la glose, c'est-à-dire vers lons, alors que le passage est omis dans AGfr II. Seule la traduction d'AGfr I peut donc être considérée comme correcte, bien qu'elle soit plus vague que le texte latin.

Les deux dernières dénominations latines contiennent toutes deux l'élément punctura: aculei sive puncturae acutae (AG XXI.9) et punctura quod acus apellamus (Al 9). Les trois traducteurs du De falconibus choisissent trois façons différentes pour rendre le binôme : traduction du dernier élément dans AGfr I (pointures agues), traduction du premier élément dans AGfr III (eguilles), et traduction du binôme entier dans AGfr II (trenchesons ou pointures acuez). Le substantif pointure, qui est attesté dès le XII siècle dans le sens de "piqûre ", surtout des serpents ou des insectes ${ }^{89}$, existe toujours mais a perdu le sens médiéval. Comme il renvoie à une douleur aiguë, l'adjectif agu peut être interprété comme redondant, mais les traducteurs rendent simplement un modèle contenant déjà cet élément superflu. Dans AGfr II, les deux composants du binôme sont synonymiques, parce que trencheson signifie également "douleur très aiguë dans le ventre ». Il est dérivé $d u$ verbe trencher et attesté $d u$ XIII $^{e}$ au XVII ${ }^{e}$ siècle ${ }^{90}$. Dès 1538 , ce terme est remplacé par la forme tranchées (« coliques aiguës »), bien que celle-ci soit, en dehors de la médecine vétérinaire, maintenant considérée comme archaïque ${ }^{91}$. Comme celle d'AGfr I, la traduction d'AGfr II est cependant problématique, parce que dans le passage concerné les vers ont disparu des versions françaises, ce qui peut tromper le lecteur sur l'origine du mal, comme nous l'avons remarqué. 
Quant à punctura quod acus apellamus, le traducteur rend cette glose de deux manières, la première fois par du mal que on appelle aiguille, ensuite par jointures que nous appellons aiguille. L'élément-clé de la traduction est le nom aiguille, que nous venons d'analyser, mais dont l'emploi cette fois-ci peut être considéré comme correct, comme c'était également le cas pour la traduction d'aculeus dans AGfr III. En ce qui concerne l'ensemble de la glose, celle-ci est correcte pour la première occurrence, même si la traduction de mal pour punctura est plus vague. Par contre, dans la seconde occurrence, où le substantif punctura est rendu par jointures, le traducteur ne semble pas avoir compris le sens du passage, comme l'avait également remarqué G. Tilander ${ }^{92}$.

Analyse de la traduction

Comme il est indiqué ci-dessus, les ectoparasites pediculus et tinea ont comme traductions prototypiques peoil et teigne. Au moment où apparaissent les premières traductions vernaculaires des traités cynégétiques latins, c'est-à-dire la seconde moitié du XIII ${ }^{e}$ siècle, ces noms français sont déjà attestés depuis un siècle, et cela vaut également pour l'adjectif peoillos, dont l'utilisation peut simplement être liée à un souci stylistique de la part du traducteur. Il ne faut donc pas s'étonner que ces termes apparaissent dans toutes les traductions qui traitent des ectoparasites ${ }^{93}$. Mais ils sont parfois accompagnés d'autres mots ou traduits sous une forme différente, ce qui nécessite quelques commentaires supplémentaires. Ainsi, pediculous (Dfr I) doit être considéré comme un latinisme pour peoillos. En outre, toujours dans Dfr I, la table contient encore la forme latine tinea, bien que le traducteur connaisse le terme français, comme il le montre par une occurrence ultérieure. Une traduction divergente se présente dans Pfr, où le traducteur rend correctement tinea par migne (3 occurrences). En revanche, l'emploi de lentes pour tinea (AGfr I) est incorrect, parce qu'il s'agit de parasites différents. Une autre confusion se trouve dans AGfr II, où le nom tinea est d'abord rendu par la paraphrase ung ver que on apelle tynia et ensuite par vers que l'on appelle tynes. Mfr contient encore une autre paraphrase, parce que enfermité de peoil y rend pediculus ${ }^{94}$. Enfin, notre corpus présente encore quelques traductions hyperonymiques, c'est-à-dire que la version française offre une terminologie plus large que le texte latin. Concrètement, il s'agit de poes ou telle vermine et ensuite vermine seul pour pediculus (AGfr II) et du trinôme taigne, rougne ou autre infection sur le cuir pour tinea (AGfrm). Ces traductions hyperonymiques ne sont cependant pas obligatoirement incorrectes, étant donné que les noms latins cachent parfois plusieurs types de parasites sous une seule dénomination ${ }^{95}$. Pour l'ensemble des occurrences, cela donne les résultats suivants ${ }^{96}$ : 


\begin{tabular}{|c|c|c|c|c|c|c|c|c|c|c|c|}
\hline & \multirow{2}{*}{$\begin{array}{l}\text { Trad. } \\
\text { prot. }\end{array}$} & \multicolumn{9}{|c|}{ Traduction non prototypique } & \multirow{2}{*}{ Total } \\
\hline & & $\begin{array}{c}\text { fr. } \\
\text { corr. }\end{array}$ & $\begin{array}{c}\text { fr } \\
\text { incorr. }\end{array}$ & latin & Latinisme & $\begin{array}{l}\text { Par: } \\
\text { lat. }\end{array}$ & $\begin{array}{c}\text { rase } \\
\text { fr. }\end{array}$ & $\begin{array}{l}\text { Hypero } \\
1 \text { él. } \\
\end{array}$ & $\begin{array}{l}\text { nyr } \\
2\end{array}$ & & \\
\hline AGfr 1 & 13 & 0 & 2 & 0 & 0 & 0 & 0 & 0 & 0 & 0 & 15 \\
\hline AGtr II & 4 & 0 & 0 & 0 & 0 & 1 & 1 & 1 & 1 & 0 & 8 \\
\hline AGfrm & 9 & 0 & 0 & 0 & 0 & 0 & 0 & 0 & 0 & 1 & 10 \\
\hline AGfr ill & 15 & 0 & 0 & 0 & 0 & 0 & 0 & 0 & 0 & 0 & 15 \\
\hline Defr 1 & 1 & 0 & 0 & 1 & 1 & 0 & 0 & 0 & 0 & 0 & 3 \\
\hline Dfr II & 4 & 0 & 0 & 0 & 0 & 0 & 0 & 0 & 0 & 0 & 4 \\
\hline Ghfr & 10 & 0 & 0 & 0 & 0 & 0 & 0 & 0 & 0 & 0 & 10 \\
\hline Gufr & 3 & 0 & 0 & 0 & 0 & 0 & 0 & 0 & 0 & 0 & 3 \\
\hline Mfr & 20 & 0 & 0 & 0 & 0 & 0 & 1 & 0 & 0 & 0 & 21 \\
\hline Pfr & 5 & 3 & 0 & 0 & 0 & 0 & 0 & 0 & 0 & 0 & 8 \\
\hline Total & 84 & 3 & 2 & 1 & 1 & 1 & 2 & 1 & 1 & 1 & 97 \\
\hline
\end{tabular}

[Trad. prot. = « traduction prototypique », $c f$. n. 28.]

7 Le schéma montre que pour désigner les ectoparasites, les traducteurs ont surtout employé ce que nous avons appelé les traductions prototypiques, c'est-à-dire peoil, peoillos et teigne. En effet, un de ces trois termes a été utilisé comme traduction unique dans 84 cas sur 97 , soit dans $86,6 \%$. En outre, les autres traductions ne sont pas forcément incorrectes ou difficiles à comprendre. Les seuls cas problématiques sont le terme latin (1), le nom français incorrect (2) et les paraphrases confondant les vers et les teignes (2), dont la première a gardé tinea, auxquels on peut éventuellement ajouter le latinisme (1), soit au maximum six occurrences ou $6,2 \%$ du total.

L'emploi élevé des traductions prototypiques simples indique également que les traducteurs ont peu recouru à des mécanismes de traduction spécifiques. En effet, ceux-ci se présentent seulement dans AGfr II (deux paraphrases et un binôme), AGfrm (un trinôme) et Mfr (une paraphrase). Dans le dernier cas, il est cependant difficile de se prononcer, parce qu'il n'est pas exclu que le modèle latin ait également contenu une paraphrase, comme c'est sans doute le cas pour les autres paraphrases qui figurent dans cette traduction. Quant aux mécanismes employés dans les traductions du $D e$ falconibus (AGfr II et AGfrm), les paraphrases semblent indiquer une certaine hésitation du traducteur en ce qui concerne la nature exacte des teignes, bien que le nom français soit déjà attesté depuis trois siècles à l'époque de ces traductions. D'autre part, les polynômes insistent plutôt sur le fait que les noms latins ne doivent pas nécessairement être compris au sens strict, mais peuvent désigner différents types d'ectoparasites.

En ce qui concerne les endoparasites, commençons par les traductions des noms généraux lumbricus et vermis, auxquels nous lions lombric et ver comme leurs deux traductions prototypiques ${ }^{97}$. Ces substantifs français sont attestés depuis le XII siècle au moins, mais avec le sens de "larve " ou de "ver terrestre ", alors que d'après les données des dictionnaires ${ }^{98}$, il n'existait pas d'occurrences de "ver intestinal » à l'époque où apparaissent les premières traductions cynégétiques. Daniel Delau, le traducteur de Ghfr et de Mfr, pourrait être le premier auteur à utiliser ces deux noms avec le sens de "ver intestinal ». Dans Ghfr, il recourt même une seule fois au binôme de toutez les lombrices et de toutez verms, mais comme il s'agit de la dernière occurrence 
de lombrices (54.6), le binôme ne sert pas à introduire un nouveau sens ${ }^{99}$. En revanche, la nouveauté de la terminologie pourrait expliquer pourquoi le traducteur de Dfr I et Gufr I, en 1284, conserve le nom latin lumbricus. Plus tard, les traducteurs recourent en général à ver, qui semble donc s'imposer. Les exceptions sont en effet rares : filandre dans AGfr III, ce qui constitue une traduction acceptable, contrairement à esguille dans le même texte et la paraphrase métonymique flux de ventre qui le blesse dans AGfr II. Ce que l'on peut schématiser sous la forme du tableau ci-contre.

58 occurrences sur 64 , soit $90,6 \%$ des cas, peuvent être considérées comme prototypiques, même si ce chiffre doit surtout être attribué à Ghfr et Mfr, qui représentent ensemble 50 occurrences des 64 , soit $78,1 \%$. En effet, pour les huit autres textes, le nombre de traductions prototypiques est seulement $8 / 14$, soit $57,1 \%$. Toutefois, même dans ces cas, et comme pour les teignes et les poux, nous constatons que les traductions erronées et les mécanismes de traduction sont peu nombreux.

\begin{tabular}{|c|c|c|c|c|c|c|c|c|}
\hline & \multicolumn{3}{|c|}{ Traduction prototypique } & \multicolumn{4}{|c|}{ Traduction non prototypique } & \multirow{3}{*}{ Total } \\
\hline & \multirow{2}{*}{ Ver } & \multirow{2}{*}{ Lombric } & \multirow{2}{*}{$\begin{array}{l}\text { Lombrics } \\
\text { et vers }\end{array}$} & \multicolumn{2}{|c|}{ Nom simple } & \multirow{2}{*}{$\begin{array}{l}\text { Nom } \\
\text { latin }\end{array}$} & \multirow{2}{*}{$\begin{array}{l}\text { Paraphr } \\
\text { méton. }\end{array}$} & \\
\hline & & & & correct & incorrect & & & \\
\hline AGfr 1 & 3 & 0 & 0 & 0 & 0 & 0 & 0 & 3 \\
\hline AGfr II & 0 & 0 & 0 & 0 & 0 & 0 & 1 & 1 \\
\hline AGfrm & 2 & 0 & 0 & 0 & 0 & 0 & 0 & 2 \\
\hline AGfr III & 0 & 0 & 0 & 1 & 2 & 0 & 0 & 3 \\
\hline Dfr I & 0 & 0 & 0 & 0 & 0 & 1 & 0 & I \\
\hline Dfr 11 & 2 & 0 & 0 & 0 & 0 & 0 & 0 & 2 \\
\hline Ghfr & 4 & 8 & 1 & 0 & 0 & 0 & 0 & 13 \\
\hline Gufr & 0 & 0 & 0 & 0 & 0 & 1 & 0 & 1 \\
\hline Mfr & 28 & 9 & 0 & 0 & 0 & 0 & 0 & 37 \\
\hline Pfr & 1 & 0 & 0 & 0 & 0 & 0 & 0 & 1 \\
\hline Total & 40 & 17 & 1 & 1 & 2 & 2 & 1 & 64 \\
\hline
\end{tabular}

Pour les traductions des dénominations complexes, il est impossible de tirer des conclusions générales, puisqu'elles ne se trouvent que dans un seul texte latin de notre corpus. Rappelons seulement que dans les trois cas, il s'agit de termes latins qui sont peu fréquents avec ce sens et qui, pour cette raison, sont accompagnés d'une explication. Mais, malgré celle-ci, ces noms ont manifestement posé des problèmes aux traducteurs, puisqu'aucune des trois dénominations n'est rendue correctement. Pour le binôme aculei sive puncturae acutae, AGfr III ne reprend que le premier élément, mais AGfr I et II insistent sur le second élément et omettent de cette façon tout rapport avec les vers. Dans anguillae, hoc est lumbrici longi, c'est surtout le nom anguilla qui a posé problème, vu que la traduction aiguille, qui se présente dans deux versions françaises, est incorrecte, et que dans une troisième traduction cette occurrence est omise. La seule traduction valable a résolu le problème terminologique en ne reprenant que la glose (vers lons). Enfin, de la séquence punctura quod acus apellamus, l'élément-clé acus est cette fois-ci correctement rendu par aiguille, mais ici c'est punctura qui est problématique. Ce substantif est d'abord traduit par mal, ce qui peut être recevable, mais ensuite par jointure, ce qui montre que le traducteur n'a pas compris le passage en question. Sur les neuf occurrences latines des dénominations complexes, nous n'avons donc que trois traductions correctes, cinq traductions incorrectes et une omission. Les tentatives des auteurs latins pour introduire des termes spéciaux ne semblent donc pas 
avoir réussi, ce qui confirme que, tout au long du Moyen Âge, il règne une grande confusion en ce qui concerne l'identification exacte des (endo-)parasites.

Conclusions

Nous avons commencé notre étude par une analyse du vocabulaire latin. Pour les ectoparasites, la situation était claire, étant donné que la terminologie se limitait à pediculus et tinea, à comprendre au sens strict ou au sens plus large d'ectoparasites en général. La dénomination pour les endoparasites présentait une variété plus grande. Nous y avons distingué deux groupes différents : d'une part les termes généraux vermis et lumbricus, d'autre part aculeus, acus et anguilla, qui à l'intérieur d'une séquence plus large, dont deux fois avec punctura, désignaient probablement des types de vers plus spécifiques.

Notre but était d'examiner si les traductions étaient correctes et si, en outre, elles ont joué un rôle dans le développement lexical à l'intérieur du champ sémantique des parasites et comment les traducteurs ont résolu d'éventuels problèmes terminologiques. En ce qui concerne le degré d'exactitude, le schéma suivant indique les traductions qui peuvent être considérées comme correctes pour les termes latins pris individuellement :

\begin{tabular}{|c|c|c|c|}
\hline & Traduction correcte ${ }^{100}$ & Traduction incorrecte & $\begin{array}{l}\text { Maintien du } \\
\text { latin }\end{array}$ \\
\hline Pediculus & $\begin{array}{l}\text { Peoil (AGfr I, AGfr II, AGfrm, Agfr III, } \\
\text { Ghfr, Mfr, Pfr) } \\
\text { Peoillos (AGfr I, Dfr I, Dfr II, Gufr I, } \\
\text { Pfr) } \\
\text { Vermine (AGfr II) }\end{array}$ & & \\
\hline Tinea & $\begin{array}{l}\text { Teigne (AGfr I, AGfr II, AGfrm, Agfr } \\
\text { III, Dfr I, Dfr II, Gufr I) } \\
\text { Teigne, rougne (...) (AGfrm) } \\
\text { Migne (Pfr) }\end{array}$ & $\begin{array}{l}\text { Lente (AGfr I) } \\
\text { Ver qui s'appelle teigne } \\
\text { (AGfr II) }\end{array}$ & Dfr I \\
\hline $\begin{array}{l}\text { Vermis / } \\
\text { Lumbricus }\end{array}$ & $\begin{array}{l}\text { Ver (AGfr I, AGfrm, Agfr III, Dfr II, } \\
\text { Ghfr, Mfr, Pfr) } \\
\text { Lombrice (Ghfr, Mfr) } \\
\text { Filandre (AGfr III) }\end{array}$ & $\begin{array}{l}\text { Flux de ventre (AGfr II) } \\
\text { Aiguille (AGfr III) }\end{array}$ & $\begin{array}{l}\text { Dfr I } \\
\text { Gufr I }\end{array}$ \\
\hline Acus / Aculeus & Aiguille (AGfr III, Alfr) & Trencheson (AGfr II) & \\
\hline Anguilla & & $\begin{array}{l}\text { Aiguille (AGfrm, AGfr } \\
\text { III) }\end{array}$ & \\
\hline (Punctura) & $\begin{array}{l}\text { Pointure (AGfr I, AGfr II) } \\
\text { Mal (Al) }\end{array}$ & Jointure (Al) & \\
\hline
\end{tabular}


34 toujours rendu fidèlement, contrairement à anguilla, qui ne l'est jamais. Pour les autres noms, les traductions exactes sont plus fréquentes que les traductions incorrectes, aussi bien en nombre de noms qu'en nombre de textes. Ces traductions incorrectes sont généralement dues à la confusion entre différents parasites, ou entre les maladies et les symptômes, le seul cas où le traducteur s'est complètement trompé étant Alexander medicus, où jointure rend punctura. Étant donné la taille microscopique de plusieurs parasites, il ne faut pas s'étonner qu'une certaine confusion règne quant à la nature exacte des organismes correspondant aux différents noms.

35

les ectoparasites, la situation semble à nouveau assez claire. Les termes français peoil, peoillos et teigne sont attestés dès le $\mathrm{XII}^{\mathrm{e}}$ siècle avec le sens qu'ils revêtent dans les textes de notre corpus et les traducteurs se sont donc en grande majorité servis de lexèmes existants. Leur apport au développement du lexique concernant les ectoparasites semble donc négligeable. Les autres noms employés par certains traducteurs, c'est-àdire lente (AGfr I), vermine (AGfr II), rogne (AGfrm), infection (AGfrm) et migne (Pfr), sont également attestés avec le sens qu'ils occupent dans notre corpus depuis au moins un siècle avant la rédaction du texte dans lequel nous les avons relevés. Le seul élément qui mérite d'être mentionné est l'emploi des polynômes par lesquels les traducteurs insistent sur le fait que le sens des noms latins n'est pas forcément restreint au parasite en question, mais peut en désigner plusieurs.

36

La situation est différente en ce qui concerne les endoparasites, puisque, selon les données fournies par les dictionnaires et les glossaires, il n'existait pas encore de terme vernaculaire pour désigner le ver intestinal à l'époque où apparaissent les premiers traités cynégétiques français. C'est donc à Daniel Delau, traducteur de Ghfr et de Mfr, et à ses confrères, qu'il est revenu de trouver une telle désignation. La solution employée par Daniel Delau est simple : soit verm, soit lombrice. Ces deux mots existaient déjà en français, mais avec le sens de "ver de terre", et dans les traductions de Delau, ils peuvent être caractérisés comme des néologismes sémantiques latinisants ${ }^{102}$. La raison pour laquelle le traducteur emploie tantôt verm, tantôt lombrice, peut être liée à une alternance des formes vermis et lumbricus dans le texte latin, mais comme les manuscrits présentent des variantes et que nous ne connaissons pas le modèle exact employé par le traducteur, il est impossible de se prononcer. Les traducteurs postérieurs ont généralement suivi l'exemple de Daniel Delau, en tout cas en ce qui concerne l'emploi de ver. Toutefois, d'autres dénominations voient également le jour, en premier lieu aiguille et filandre, deux noms qui apparaissent dans le troisième quart du XIve siècle, le premier chez Gace de la Buigne, le second chez Henri de Ferrières, et qui sont seulement employés pour les oiseaux rapaces. Actuellement, H. Beaufrère définit les aguilles comme « ancien terme pour désigner des "vers microscopiques qui se logent sous la peau des oiseaux [...]" " ${ }^{103}$ et il ajoute que la nature exacte de ces parasites est difficile à déterminer, bien qu'une identification avec la larva migrans ne soit pas exclue. Pour les filandres, il note que ce sont des « vers parasites que l'on trouve dans les émeuts [...], vraisemblablement des capillaires" ${ }^{104}$. Il est cependant improbable qu'à l'époque médiévale on ait déjà pu établir une distinction pareille, et les deux substantifs français peuvent donc être interprétés comme des synonymes. Le traducteur d'Alexander medicus se trouve probablement parmi les premiers utilisateurs d'aiguille ${ }^{105}$. Il est toutefois évident que ce n'est pas cette traduction qui est responsable

Médiévales, 51 | automne 2006 
de la diffusion du terme, mais Gace de la Buigne. Les trois dernières dénominations, à savoir pointure, trencheson et flux de ventre, sont toutes attestées dès le $\mathrm{XII}^{\mathrm{e}}$ ou le $\mathrm{XIII}^{\mathrm{e}}$ siècle, donc au moins deux siècles avant leur utilisation dans notre corpus. Les traducteurs ont certes pu jouer un rôle dans la diffusion de ver au sens de «ver intestinal ", mais il s'agit d'un terme fréquent par ailleurs, également en dehors du présent corpus, ce qui vaut dans une moindre mesure aussi pour lombric. Au total, pour l'ensemble du vocabulaire étudié, l'apport des traducteurs des traités cynégétiques semble donc assez modeste, étant donné qu'ils utilisent surtout des termes attestés de longue date et donc déjà bien intégrés dans le lexique français.

Annexe : glossaire

37 (Les termes latins sont suivis d'un astérisque.)

aculeus* : aiguille, ver intestinal

acus* : aiguille, ver intestinal

aiguille : petit ver intestinal, peut-être la larva migrans

anguila* : aiguille, ver intestinal, probablement du type des Ascaridia

ectoparasites : parasites qui se trouvent à l'extérieur du corps

endoparasites : vers intestinaux qui se trouvent à l'intérieur du corps

filandre: ver intestinal qu'on trouve dans les émeuts, probablement des capillaires

lente : œuf des poux

lombrice : ver intestinal

lumbricus* : ver intestinal (vocabulaire plus spécialisé)

migne : mite, ver rongeur

pediculus* : pou ou plus généralement, ectoparasites

pointure : piqûre, surtout des serpents ou des insectes

pou : pou

punctura* : point de côté

rogne : gale invétérée

teigne : teigne

tinea* : teigne, plus généralement, ectoparasites

trencheson: douleur très aiguë dans le ventre

ver: ver intestinal, parasite en forme de ver qui se développe dans le corps de l'homme et des animaux

vermine : vermine, l'ensemble des insectes parasites

vermis* : ver intestinal (vocabulaire général)

[Cet article s'inscrit dans un mandat post-doctoral consacré à la terminologie médicale dans les traductions des traités cynégétiques latins, subventionné par le conseil de recherche (Onderzoeksraad) de la K.U. Leuven (PDM/04/49).]

\section{NOTES}

1. Pour ne citer que quelques publications : L. A. SHORE, «A Case Study in medieval nonliterary Translation : scientific Texts from Latin to French », dans J. BEER éd., 
Medieval Translators and their Craft, Kalamazoo, 1989 (Studies in Medieval Culture, 25), p. 297-327 ; J. Ducos, « Traduction et autorité. Le cas des Météorologiques d'Aristote », Bien dire et bien aprandre, 14, 1996, p. 207-218 ; M.-D. GLESSGEN, Die Falkenheilkunde des " Moamin » im Spiegel ihrer volgarizzamenti. Studien zur Romania Arabica, Tübingen, 1996 (Beihefte zur Zeitschrift für romanische Philologie, 269-270), et M. GoYENS, « Le développement du lexique scientifique français et la traduction des Problèmes d'Aristote par Evrart de Conty (ca 1380) », Thélème, 189, 2003, p. 189-207.

2. Dans les textes de fauconnerie latins, teignes et poux sont même les maux les plus fréquents ; $c f$. B. VAN DEN ABEELE, La fauconnerie au Moyen Âge. Connaissance, affaitage et médecine des oiseaux de chasse d'après les traités latins, Paris, 1994 (Sapience, 10), p. 183. Aucune étude semblable n'a été menée pour l'ensemble des traités cynégétiques en moyen français, mais la fréquentation régulière de ces textes nous apprend que des remèdes contre les parasites y sont également courants.

3. Les parasites ne figurant pas dans les traductions françaises des traités d'Adélard de Bath (omission du paragraphe 18 dans Afr II), de Frédéric II, de Gerardus falconarius (omission du chap. 8) et de Robert du Herlin, ni dans la seconde traduction de Guillelmus falconarius, nous n'avons pas intégré ces textes dans notre corpus.

4. Nous reprenons les sigles de VAN DEN ABEELE, La fauconnerie du Moyen Âge, op. cit., p. 23-30.

5. Textes édités dans A. SMETS, Des faucons : les quatre traductions en moyen français du De falconibus d'Albert le Grand. Analyse lexicale d'un dossier inédit, thèse de doctorat, K.U. Leuven, 2003, vol. II. L'édition des quatre traductions paraîtra l'an prochain dans la série Bibliotheca cynegetica chez l'éditeur J. Laget.

6. Sources inédites des Auzels Cassadors de Daude de Pradas. Grisofus Medicus. Alexander Medicus. Deux traités latins de fauconnerie du XII siècle publiés avec des traductions en vieil italien de Grisofus et une traduction en vieux français d'Alexander, G. TILANDER éd., Lund, 1964 (Cynegetica, 10).

7. Traductions en vieux français de Dancus rex et Guillelmus falconarius, G. TILANDER éd., Karlshamn, 1965 (Cynegetica, 12).

8. Moamin et Ghatrif : traités de fauconnerie et des chiens de chasse. Édition princeps de la version franco-italienne, avec 3 planches hors texte, H. TJERNELD éd., Stockholm-Paris, 1945 (Studia romanica holmiensia, 1).

9. Pour la référence, voir la n. 8 .

10. Moamin et Ghatrif, éd. cit.

11. Ms. Lyon, BM, 765 , fo $180 \mathrm{v}^{\circ}-186$ (inédit).

12. Pour plus d'informations sur la littérature cynégétique, voir VAN DEN ABEELE, La fauconnerie au Moyen Âge, op. cit., ID., La littérature cynégétique, Turnhout, 1996 (Typologie des sources du Moyen Âge occidental, 75) et A. SMETS, B. VAN DEN ABEELE, « Manuscrits et traités de chasse français du Moyen Âge. Recensement et perspectives de recherche ", Romania, 116 3/4, 1998, p. 316-367.

13. Voir aussi le Dictionnaire des Lettres françaises, le Moyen Âge, éd. revue et dirigée par G. HASENOHR et M. ZINK, Paris, 1992, p. 45 et 366-367.

14. Dès avant 1241 pour Moamin, traduit d'abord par le médecin Théodore ; ibid., p. 1020 .

15. Sur la fréquence des poux dans les traités cynégétiques médiévaux, voir VAN DEN ABEELE, La fauconnerie au Moyen Âge, op. cit., p. 185 et GLESSGEN, Die Falkenheilkunde des "Moamin », op. cit., II, p. 626-627. 
16. Pour souligner l'aspect nuisible, Dancus rex parle de tineam malam (Dancus rex, Guillelmus falconarius, Gerardus falconarius. Les plus anciens traités de fauconnerie de l'Occident publiés d'après tous les manuscrits connus, G. TILANDER éd., Lund, 1963, p. 78), ce qui est devenu mal(l)e taigne dans Dfr II (Traductions en vieux français de Dancus rex, éd. cit., p. 36).

17. GLESSGEN, op. cit., II, p. 626.

18. A. ERnoUt, A. MEILLET, Dictionnaire étymologique de la langue latine. Histoire des mots (1932), Paris, $1959^{4}$, p. 493.

19. Ms. cité du Vatican, Biblioteca Apostolica Vaticana (BAV), Reg. lat. 1617.

20. VAN DEN ABEELE, op. cit., p. 184.

21. ERNOUT, MEILLET, op. cit., p. 692.

22. VAN DEN ABEELE, op. cit., p. 186. Pour plus d'informations sur la présence des vers et la terminologie dans la littérature médicale antique, voir V. NicLOT, « Les vers intestinaux dans la littérature médicale antique », Paris médical : la semaine du clinicien, LXIV, 1927, p. 291-299.

23. VAN DEN ABEELE, op. cit., p. 187.

24. ERNOUT, MEILlET, op. cit., p. 369, et R. E. LATHAM, Dictionary of medieval Latin from British sources, Londres-Oxford, 1975-, I, p. 1658.

25. ERNOUT, MEILLET, op. cit., p. 724, et A. WALDE, Lateinisches etymologisches Wörterbuch, Heidelberg, $1965^{4}$, II, p. 760.

26. J. N. ADAMS, Pelagonius and Latin Veterinary Terminology in the Roman Empire, LeydeNew York-Cologne, 1995, p. 325 et 560 (Studies in ancient medicine, 11).

27. Le nom lumbricus n'est pas du tout mentionné par J. N. ADAMS.

28. Selon la sémantique du prototype, il faut distinguer les éléments centraux, comme ici lumbricus et vermis, qui sont clairement les termes les plus fréquents, des éléments périphériques, comme ici acus, aculeus et angula, qui peuvent désigner la même notion mais connaissent un emploi plus marginal. Cette théorie est largement décrite dans différents ouvrages de D. GEERAERTS (par exemple Diachronic Prototype Semantics. A contribution to Historical Lexicology, Oxford, 1997, Oxford Studies in Lexicography and Lexicology). Les données essentielles sont également reprises en français par W. DE Mulder (« La linguistique diachronique, les études sur la grammaticalisation et la sémantique du prototype : présentation ", Langue française, 130, 2000, p. 8-32, ici p. 19-27).

29. ERNOUT, MEILLET, op. cit., p. 724 et 369, définissent vermis comme « ver » et lumbricus comme « ver de terre » ou, par analogie, « ver intestinal », tout comme WALDE, op. cit., II, p. 760 et I, p. 831, et Le Grand Gaffiot. Dictionnaire latin-français. Nouvelle édition revue et augmentée, dir. P. FLOBERT, Paris, 2000, p. 1689 et 935. La définition que donne le Oxford latin dictionary (Oxford, 1968-1982, II, p. 2037 et p. 1049) pour vermis est encore plus large, à savoir « a worm, maggot or other small creature of similar appearance », alors que la définition de lumbricus y est identique à celle des autres dictionnaires. Seul R. E. LATHAM, Revised medieval latin word-list from British and Irish sources, Londres-Oxford, 1965 , p. 508 et 282, ne suit pas ce schéma : vermis y est défini comme "serpent, Diable » et lumbricus comme « ver ». Plusieurs autres dictionnaires (R. E. LATHAM, Dictionary of medieval Latin, op. cit. ; Mittellateinisches Wörterbuch bis zum ausgehenden 13. Jahrhundert, Munich, 1967- (dorénavant MLW) ; J. F. NIERMEYER, C. VAN DE KIEFT, Mediae latinitatis lexicon minus, éd. remaniée par J. W. J. BURGERS, Leyde-Boston, $2002^{2}$, et Thesaurus linguae 
latinae, Leipzig-Munich, 1900-) ne contiennent pas (encore) les deux termes en question et ne sont donc pas intégrés dans cette comparaison.

30. Parmi les auteurs cités figurent Plaute (ERnout, Meillet, op. cit., p. 369) et Caton (ibid., et Oxford latin dictionary, op. cit., II, p. 1049).

31. LATHAM, Dictionary of medieval Latin, op. cit., I, p. 1658.

32. Cf. J. L. G. MowAT (éd.), Alphita. A medico-botanical Glossary from the Bodleian manuscript Selden B. 35, Oxford, 1887.

33. Il faudrait étendre le corpus pour vérifier si ces observations se confirment, mais cela dépasse les limites de la présente contribution. Nous avons cependant fait un premier sondage pour les termes vermis et lumbricus dans les textes latins d'origine italienne du Moyen Âge (http://www.uan.it/alim) : nous avons ainsi obtenu 39 résultats pour vermis chez une dizaine d'auteurs, dont Joachim de Flore, Bernard de Clairvaux et Guillelmus falconarius. Nous n'avons en revanche obtenu que six résultats pour lumbricus : trois dans Dancus rex et trois dans Guillelmus falconarius.

34. Thesaurus linguae latinae, op. cit., IIa, p. 50.

35. Dancus rex, Guillelmus falconarius, Gerardus falconarius, éd. cit.; chap. 8 ; p. 210 et 233 (à moins que la version de Gerardus utilisée par Albert le Grand ne présente la variante anguilla pour angulia, $c f . \mathrm{ms}$. A).

36. Mittellateinisches Wörterbuch, op. cit., I, p. 643.

37. VAN DEN ABEELE, op. cit., p. 187, cf. C. BOUGEROL, Essai sur la pathologie des oiseaux de chasse au vol, thèse pour le doctorat vétérinaire, Maisons-Alfort, 1967, p. 44.

38. Cf. MLW, I, p. 643.

39. ERNOUt, MeILlet, op. cit., p. 5.

40. Ibid.

41. P. IMBS, B. QUEMADA dir., Trésor de la langue française, Nancy-Paris, 1971-1994 (dorénavant TLF), II, p. 296, et H. BEAUfRÈRE, Lexique de la chasse au vol. Terminologie française du XVI e au XXe siècle, Nogent-le-Roi, 2004, p. 12.

42. B. VAN DEN ABEeLE signale qu'acus apparaît également comme un nom de maladie dans le pseudo-Hippocrate, mais semble y désigner un autre mal (op. cit., p. 187). 43. Adelard of BATH, Conversations with his Nephew. On the Same and the Different, Questions on Natural Science and On birds, éd. et trad. CH. BURNETT, coll. I. RONCA, P. Mantas España, B. Van den Abeele, Cambridge, 1998 (Cambridge Medieval Classics 9), chap. 18, p. 258 et 272 ; cf. LATHAM, Dictionary of medieval Latin, op. cit., I, p. 23. Rappelons que les traductions françaises du traité d'Adélard de Bath ne reprennent pas le passage en question (cf. la n. 3).

44. Chap. 8 dans B. VAN DEN ABEELE, Les traités de fauconnerie latins au Moyen Âge, thèse de doctorat, Louvain-la-Neuve, 1991, annexes p. 206 ; cf. ID., La fauconnerie au Moyen Âge, op. cit., p. 187.

45. Le MLW, I, p. 141, mentionne comme seul exemple le De falconibus et sa définition est peu précise, parce qu'il explique cette occurrence comme une maladie des autours causée par des piqûres.

46. ERNOUT, MEILLET, op. cit., p. 546.

47. W. F. RichaRDSon, A Word Index to Celsus : De medecina, Auckland, 1982.

48. Du CANGE, Glossarium mediae et infimae latinitatis, Niort, 1840-1850, VI, p. 571.

49. BOUGEROL, op. cit., p. 35-36, mentionne parmi les symptômes de l'aspergillose, " peut-être la cause la plus fréquente de maladie et de mortalité chez nos rapaces 
captifs ", des problèmes de respiration, qui peut être alors légèrement accélérée et difficile, comme en cas de points de côté.

50. Pour la notion de terme prototypique, ou ici de traduction prototypique, voir la n. 28.

51. peoil dans Ghfr et Mfr, peus dans Gufr I, poes dans AGfr II, poils dans AGfr I, poilz et poix dans AGfr III, poouil dans Gufr I, pos dans AGfr III, pouez dans AGFrm, pous dans AGfr I, AGfr II et Pfr et poux également dans Pfr.

52. W. VON WARTBURG, Französisches Etymologisches Wörterbuch. Eine Darstellung des galloromanischen Sprachschatzes, Bonn-Bâle, 1922-, VIII, p. 148, dorénavant FEW.

53. Le ms. T est le codex New Haven, Beinecke Library, 446.

54. poulleux dans Dfr II, pouilleus et pouilleux dans respectivement Gufr I et Pfr, pouil(l)ious dans AGfr I, pouilloux dans Dfr II ou encore le latinisme pediculous dans Dfr I. D'autres formes avec $<\mathrm{d}>$ ne figurent ni dans la Base de lexiques du moyen français de l'ATILF/Équipe « Moyen français et français préclassique » (site web www.atilf.fr, dorénavant BLMF), ni dans le FEW, VIII, p. 150, ni dans F. GODEFROY, Dictionnaire de l'ancienne langue française et de tous ses dialectes $d u I^{e}{ }^{e}$ au XV $V^{e}$ siècle (1881-1902), 10 vol., rééd. Paris-Nendeln, 1969, X, p. 377, ni dans A. TOBLER, E. LOMMATZSCH, Altfranzösisches Wörterbuch, Berlin-Wiesbaden-Stuttgart, 1925-1965, rééd. 2002 (dorénavant TL), VII, col. 688.

55. FEW, VIII, p. 150.

56. À savoir une catégorie générale regroupant des sous-catégories appelées hyponymes : « autour » est un hyponyme de « rapace », par exemple (NdlR).

57. Ibid., XIV, p. 292 et TLF, XVI, p. 1041.

58. TLF, XV, p. 1439. Selon le FEW, XIII 1 , p. 340, ce terme date du XIII ${ }^{e}$ siècle.

59. BEAUFRÈRE, op. cit., p. 348-349. Voir à ce sujet également Bougerol, op. cit., p. 33 ; VAN DEN ABEELE, La fauconnerie au Moyen Âge, op. cit., p. 184, et GLESSGEN, op. cit., II, p. 646-647.

60. Bien que cette paraphrase soit incorrecte, elle existe de longue date, comme en témoigne l'exemple suivant tiré du commentaire du Carmen paschale de Sedulius par Remi d'Auxerre ( $\mathrm{x}^{\mathrm{e}}$ siècle) : « Tinea est vermis quicquid conterit adurens (...)» (exemple cité dans C. JEUDY, « Glossaires juvénaliens du haut Moyen Âge », dans J. HAMESSE éd., Les manuscrits des lexiques et glossaires de l'Antiquité tardive à la fin du Moyen Âge, Actes du Colloque international d'Erice (23-30 septembre 1994), Louvain-la-Neuve, 1996, p. 253-282. Un autre exemple français figure dans Les lamentations de Matheolus de Jean Le Fèvre, où cette paraphrase est employée au sens figuré : « Mariage est plus fors vermine, / Que le ver qu'on appelle tine » (voir Les lamentations de Matheolus de Jean le Fèvre, de Resson, A. G. VAN HAMel éd., Paris, 1892, I, p. 57 ; II, v. 381-382). Cet exemple du XIV siècle est cité par GODEFROY, op. cit., X, p. 747, et TL, X, p. 149.

61. FEW, X, p. 469 et TLF, XIV, p. 1199.

62. FEW, IV, p. 664-665 et TLF, X, p. 175.

63. TLF, X, p. 1091. Le FEW, V, p. 250, situe la première attestation au XIII siècle.

64. Leurs dras demainjuënt les mines : $\mathrm{v} .1012$ de Sainte Leocade dans Les miracles de Nostre Dame par Gautier de Coinci, V. F. KoenIG éd., Genève-Paris, 1961, II, p. 44. Voir également O. Collet, Glossaire et index critique des cuvres d'attribution certaine de Gautier de Coinci (Vie de sainte Cristine et Miracles de Nostre Dame), Genève, 2000, p. 332.

65. TL, VI, p. 60.

66. FEW, VI 1, p. 332.

67. GODEFROY, op. cit., V, p. 336. 
68. Cf. G. TILANDER, Glanures lexicographiques, Lund-Londres-Paris-Oxford-Leipzig, 1932, p. 171, qui mentionne ici les occurrences de Pfr.

69. Ibid., p. 172 ; cf. TLF, XI, p. 894 et XV, p. 1439.

70. FEW XIV, p. 291 et TLF, XVI, p. 1008.

71. Moamin et Ghatrif, éd. cit., p. 369. Le ms. X est le codex Venise, Biblioteca di San Marco, cod. lat. VII, 24.

72. Ibid. Le ms. C est le codex Paris, BnF, lat. 11208.

73. Cf. GLESSGEN, op. cit., I, p. 272-319 et 335-395. Les recherches en cours de

S. Marruncheddu (Turin) sur les traductions françaises du Moamin nous donneront peut-être plus de détails à ce sujet.

74. Cf. GoDEFROY, op. cit., X, p. 846, cite la phrase suivante : « La char quant ele muert fait manger a[s] lumbris ». Celle-ci figure dans Le Sermon de Guischart de Beaulieu. Édition critique de tous les manuscrits connus avec introduction, A. GABRIELSON éd., Uppsala-Leipzig, 1909, v. 51, p. 6.

75. Cf. FEW, V, p. 442.

76. Cela se constate également dans des corpus plus larges. Ainsi, la base des textes en moyen français (BTMF) contient plus de cinquante occurrences de ver(s) ("ver ») pour la période médiévale, mais aucune de lombric, qui ne figure pas non plus dans la base des textes du français ancien (TFA). Voir les bases TFA (http://www.lib.uchicago.edu/ efts/ARTFL/projects/TLA/index.html) et BTMF (http://atilf.atilf.fr/dmf.htm).

77. FEW III, 535 et TLF VIII, 877.

78. Les livres du roy Modus et de la royne Ratio. Publiés avec introduction, notes et glossaire, G. TILANDER éd., Paris, 1932, chap. 107, p. 210 ; cf. TLF VIII, 877. Remarquons que les dictionnaires mentionnent explicitement qu'il s'agit d'une maladie des oiseaux de proie (cf. GODEFROY, op. cit., IX, p. 619, et TL III, col. 1845).

79. BeAUFrère, op. cit., p. 174.

80. Godefroy, op. cit., IX, p. 631 ; FEW III, p. 645 et TL III, col. 1959. Le FEW (III, p. 645) situe la première attestation de flux (dou ventre) au XIII ${ }^{\mathrm{e}}$ siècle, sans indication de source, alors que le TLF (VIII, p. 1011-1012) indique qu'il s'agit d'un emprunt de 1306. Les exemples mentionnés dans Godefroy (IX, p. 631) et TL (III, col. 1958-1959) datent tous du XIV ${ }^{e}$ siècle ou des siècles postérieurs.

81. VAN DEN ABEELE, La fauconnerie au Moyen Âge, op. cit., p. 186.

82. BOUGEROL, op. cit., p. 43-45.

83. AlBeRTUS MAGNUS, De animalibus libri XXVI. Nach der Kölner Urschrift, H. STADLER éd., Munich, 1916-1920, p. 1476 (Beiträge zur Geschichte der Philosophie des Mittelalters, 15-16) : «Si vero falco lumbricis affligitur in ventre et in egestionibus eius aliquid de talibus apparuerit».

84. A. REY dir., Dictionnaire historique de la langue française, rééd. Paris, 1998, p. 67.

85. FEW, XXIV, p. 199.

86. TLF II, 296, cf. GODEFROY, op. cit., VIIIc, p. 55 et M. LACHIVER, Dictionnaire du monde rural : les mots du passé, Paris, 1997, p. 53. G. TILANDER mentionne une occurrence d'aiguillon dans le sens de « petit ver » dans un traité de la fin du XIII ${ }^{\mathrm{e}}$ ou du début du XIV siècle (cf. Glanures lexicographiques, op. cit., p. 19).

87. BEAUFRÈre, op. cit., p. 12.

88. La confusion aurait également pu être provoquée par des erreurs de transcription ou de copie, par exemple d'un modèle latin contenant aguilla pour anguilla, suite à 
l'omission d'un tilde. Le MLW (I, 414) signale d'ailleurs une occurrence d'agullia, dans le sens de "flèche d'une église ", un des sens secondaires d'aiguille.

89. FEW IX, p. 596 et TLF XIII, p. 680.

90. FEW XIII ${ }_{2}$, p. 280-281.

91. TLF XVI, p. 490.

92. Sources inédites des Auzels Cassadors, éd. cit., p. 48.

93. Seul Afr ne mentionne pas d'ectoparasites.

94. Quand la paraphrase française rend une paraphrase latine, nous la considérons comme une traduction prototypique.

95. GlessGen, op. cit., II, p. 626.

96. Nous n'avons pas tenu compte des occurrences omises dans AGfr II, étant donné que d'autres occurrences des mots en question y sont bel et bien traduites. Par conséquent, les omissions ne sont pas dues à une carence lexicale du traducteur, mais à sa décision de ne pas reprendre certains paragraphes. Comme les termes à examiner se trouvent ailleurs, cette décision ne porte pas à conséquences pour le phénomène examiné ici.

97. Nous ne pouvons pas faire de distinction entre les traductions de lumbricus, d'une part, et celle de vermis, d'autre part, étant donné que nous ne connaissons pas la terminologie du modèle latin employé pour Gfr et Mfr.

98. Nous avons aussi comparé ces données avec quelques glossaires médiévaux qui étaient à notre disposition, dont T. HUNT, Teaching and Learning Latin in 13th century England, Cambridge, 1991, mais nous n'avons pas trouvé d'indications de ce genre dans les glossaires antérieurs à 1250 .

99. D'ailleurs, nous ne connaissons pas le modèle latin, qui peut également contenir un binôme à cet endroit.

100. Rappelons qu'une traduction exacte, prototypique ou pas, donne un équivalent correct tenant compte de tous les éléments liés au terme latin et que par conséquent, des équivalents qui se limitent par exemple aux symptômes, ne peuvent pas être considérés comme corrects.

101. Cette traduction est considérée comme incorrecte étant donné que lumbricus reprend anguilla et la traduction d'aiguille pour anguilla est incorrecte.

102. Dans le cas d'un néologisme sémantique latinisant, le traducteur donne le sens du terme latin à un terme qui est déjà attesté en français, et formellement lié au terme latin, mais ayant une autre acception en français. Sur les différents types de néologismes, voir A. MERTENS, La néologie dans la traduction par Jean d'Antioche (1282) du "De inventione » de Cicéron et de la "Rhetorica ad Herennium ». Abit - afit, mémoire de licence, K.U. Leuven, 1989, p. 51.

103. BEAUfRÈre, op. cit., p. 12.

104. Ibid., p. 174.

105. G. TILANDER situe la traduction au $\mathrm{XV}^{\mathrm{e}}$ siècle (Sources inédites des Auzels Cassadors, éd. cit., p. 46), mais le manuscrit date du XIV ${ }^{e}$ siècle. Voir SMETS, VAN DEN ABEELE, « Manuscrits et traités de chasse français du Moyen Âge. », loc. cit., p. 325. 


\section{RÉSUMÉS}

On connaît le rôle des traductions médiévales dans le développement du lexique scientifique, et l'article analyse ce phénomène pour les noms des parasites des rapaces à partir d'un corpus composé de 11 textes cynégétiques français ayant un modèle latin et traitant ce sujet. Ce choix s'explique par le fait que les parasites (ecto- ou endoparasites) sont les maux les plus fréquents dans ces traités. Dans le domaine latin, le vocabulaire se limite à tinea et pediculus pour les ectoparasites, et aux termes généraux vermis et lumbricus pour les endoparasites, complétés par quelques occurrences d'aculeus, acus et anguilla. La diversité lexicale est plus riche en français: surtout peoil et teigne pour les ectoparasites, mais aussi vermine, lente et migne, tout comme le maintien du latin, et ver, lombrice, filandre, esguille, ... pour les endoparasites. Les traducteurs utilisent surtout des termes déjà existants, mais le traducteur de Moamin semble être le premier à employer les noms ver et lombrice dans le sens de «ver ou lombric intestinal ». Enfin, il s'avère que certains termes, comme pediculus, sont toujours rendus correctement, alors que d'autres, comme anguilla, ne le sont jamais.

"Lice, Worms and Vermin »: a Semantical Study of the Names of the Birds of Prey's Parasites in Cynegetic Texts translated from Latin to French. This contribution proposes an analysis of the role played by translations in relationship to the development of scientific vocabulary in the Middle Ages. To be precise, it is a study of the names of the birds of prey's parasites, based on a corpus of the 11 texts in Old and Middle French which deal with this topic and have a Latin source text. The reason for this choice is that in hunting literature parasites (both endo- and ectoparasites) are the diseases with the highest frequency. The Latin vocabulary is limited to tinea and pediculus for the ectoparasites, and to the general terms vermis and lumbricus for the endoparasites, these being completed by a few occurrences of aculeus, acus and anguilla. The French vocabulary is more diversified : especially peoil and teigne for the ectoparasites, but also vermine, lente and migne, just as the Latin term, and ver, lombrice, filandre, esguille, ... for the endoparasites. The translators generally choose terms which already existed in French, but the translator of the Moamin seems to be the first one to use the names ver and lombrice with the meaning of "intestinal worm ». Finally, we observe that some terms, as pediculus, are always correctly translated, and others, like anguilla, never.

\section{INDEX}

Mots-clés : lexique, traduction, médecine vétérinaire, littérature cynégétique, parasites, oiseaux rapaces

Keywords : translation, Vocabulary, veterinary medecine, cynegetic literature, birds of prey

\section{AUTEUR}

\section{AN SMETS}

Fac. Letteren K.U. Leuven, Subfaculteit Taalkunde Blijde Inkomststraat 21, B-3000 Leuven 